\title{
Claw Hand Deformity: A Rare Complication of Herpes Zoster
}

\author{
Tachit Jiravichitchai ${ }^{\prime}$ \\ Waree Chira-adisai ${ }^{1}$ \\ Monratta Panuwannakorn' \\ Sasisopin Kiertiburanakul iD ${ }^{2}$ \\ 'Department of Rehabilitation Medicine, \\ Faculty of Medicine Ramathibodi \\ Hospital, Mahidol University, Bangkok, \\ Thailand; ${ }^{2}$ Division of Infectious Diseases, \\ Department of Medicine, Faculty of \\ Medicine Ramathibodi Hospital, Mahidol \\ University, Bangkok, Thailand
}

\begin{abstract}
Distal upper extremity weakness is a rare complication after herpes zoster, which can be easily misdiagnosed by other nerve entrapment syndromes. We present a $31-$ year-old immunocompromised woman who developed a claw-like deformity of her right hand after full medical treatment and resolution of herpes zoster. The electrodiagnostic finding was compatible with right multiple mononeuropathies of the median and ulnar nerves, ongoing axonal loss, unlike the nerve entrapment patterns. The early recognition and early electrodiagnosis of herpes zoster-induced distal motor weakness, especially in the upper extremity, is necessary to exclude the nerve entrapment syndrome and to be the baseline for functional motor recovery prediction. The prognosis of functional motor recovery is considered good but may take months to years to accomplish. Rehabilitation management plays an important role after full medical treatment.
\end{abstract}

Keywords: herpes zoster, claw hand deformity, electrodiagnosis, rehabilitation, hand splint

\section{Introduction}

Herpes zoster (HZ), or shingles, is the secondary stage of a common viral disease caused by the varicella-zoster virus (VZV). The primary stage usually occurs in childhood, which is also known as chickenpox. By this stage, the virus persists in the dorsal root ganglia and can reactivate later, causing HZ. Cutaneous manifestation is the main symptom of the secondary stage, which usually appears in the thoracic dermatomes. ${ }^{1,2}$ The infection commonly occurs in elderly or immunocompromised patients, and even the general population. ${ }^{1-3}$ The most common and potentially debilitating complication after $\mathrm{HZ}$ is postherpetic neuralgia (PHN), which is pain along the dermatomal distribution. ${ }^{4}$

The HZ-paresis or limb weakness is uncommon, especially in the upper extremity. ${ }^{5}$ Few cases in literature have reported on the mononeuropathy of the distal upper extremity, which commonly presents with pain and paresthesia along with suspected nerve distribution followed recently by motor weakness along with myotomal distribution, following our patient's symptoms. ${ }^{6-9}$

In this report, we present the case of an immunocompromised patient who developed a rare complication of persistent weakness in the right hand (claw hand deformity) following the resolution of $\mathrm{HZ}$.

\section{Case Report}

A 31-year-old woman, diagnosed as stage 4 diffuse large B-cell lymphoma (DLBCL) in July 2020, had been receiving treatment with intravenous
Correspondence: Tachit Jiravichitchai Department of Rehabilitation Medicine, Faculty of Medicine Ramathibodi Hospital Mahidol University, 270 Ramathibodi Hospital, Ratchathewi, Bangkok, Thailand Email tachit.jir@mahidol.ac.th; mewtechi@gmail.com 
chemotherapy consisting of rituximab, cyclophosphamide, doxorubicin, vincristine, and prednisolone (R-CHOP) combined with intrathecal methotrexate from July 2020 to January 2021 (6-month period) without previous complication. On December 16, 2020, she developed sharpshooting pain in her right hand that radiated to the right forearm. A few days after the symptom, multiple groups of vesicles appeared on the volar side of her right hand (midpalm, pisiform bony prominent area, and fifth metacarpophalangeal joint area), the dorsal side of the right wrist, and the lateral forearm and arm, including the right scapular area. The vesicles were more severe on the erythematous to violaceous bases, as shown in Figure 1.

She was diagnosed with disseminated $\mathrm{HZ}$ and admitted for empirical intravenous acyclovir treatment on December 21, 2020. She was treated intravenously for 7 days and orally for another 7 days, and the wounds improved. PHN developed during admission and was treated with 300 milligrams per day of pregabalin. Neuralgia improved within 6 weeks. She also complained about the rapid weakening of the right handgrip since admission and her hand undertook an obvious claw-like deformity in January 2021. She was sent to a physiatrist for assessment with physical and occupational therapy program prescriptions. Physical examination revealed an obvious claw-like deformity of the right hand (metacarpophalangeal (MCP) joints hyperextension and interphalangeal (IP) joint slight flexion of second to fifth digits). Marked atrophy of intrinsic muscles of the right hand was observed, especially at the first dorsal interosseous (FDI) and abductor digiti minimi (ADM) muscles. Muscle power of the right upper extremity was grade $\mathrm{V}$ for all except grade I for the right FDI muscle, grade II for the right ADM muscle, and grade IV for the right abductor pollicis brevis (APB) muscle. Grip strength of the right hand as measured by a hand dynamometer showed 1.5 kilograms. The left hand was measured at 8.6 kilograms. Deep tendon reflex was decreased at the right brachioradialis reflex. Light touch and pinprick sensations decreased mildly along with right ulnar nerve distribution. The passive range of motion for the MCP and IP joints of all digits on the right hand was normal.

Electrodiagnosis was done on March 4, 2021 for evaluation of distal neuropathy in the right hand. Right ulnar sensory nerve conduction study (NCS) showed no response, as shown in Figure 2. Right ulnar motor NCS showed normal distal motor latency, but also a marked decrease in compound motor action potential (CMAP) amplitude with mild slow motor nerve conduction velocity (NCV), as shown in Figure 3. Right median motor NCS showed normal distal motor latency and CMAP amplitude, as shown in Figure 4. The results of right radial and dorsal ulnar cutaneous NCS were normal. Needle electromyography (EMG) examination showed signs of membrane

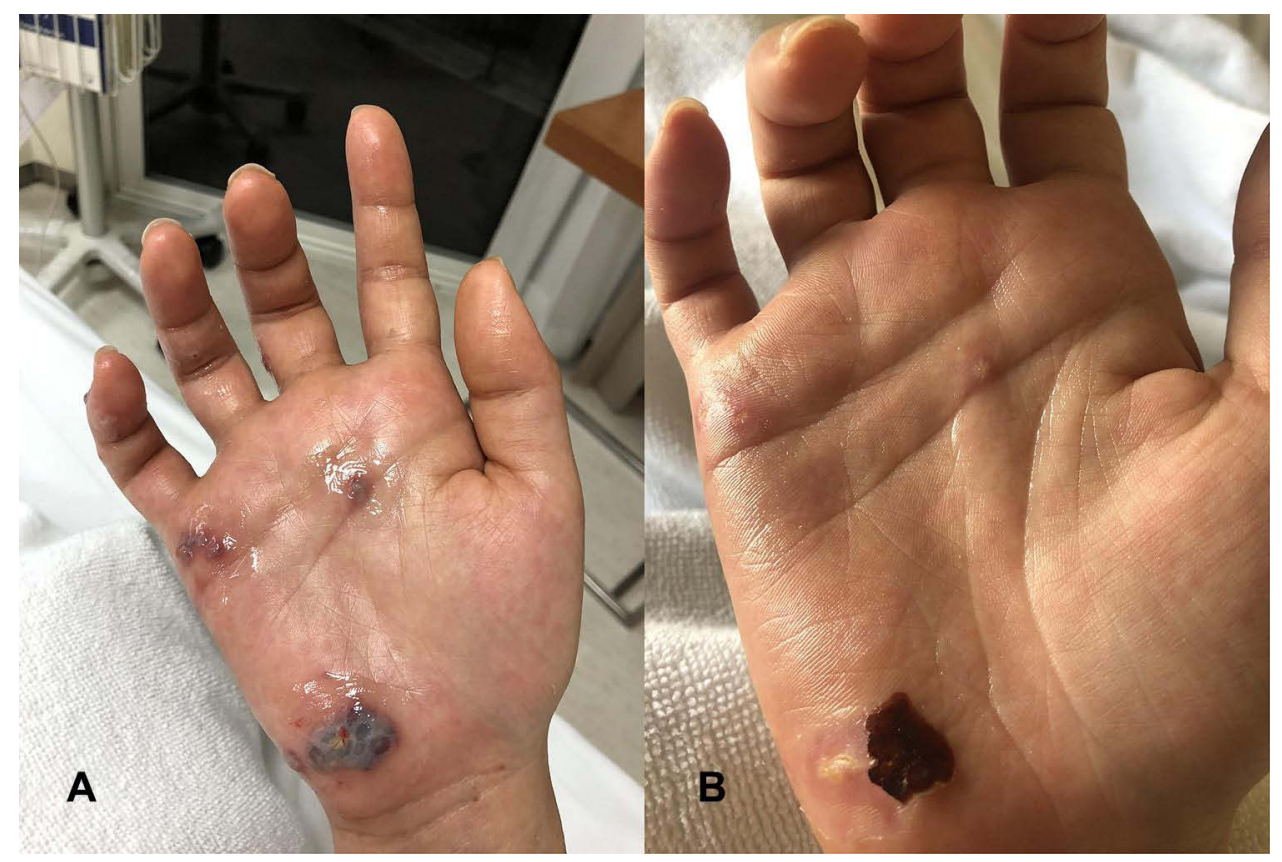

Figure I Clusters of round and polygonal vesicular lesions on erythematous to violaceous base at the right hand (A) and post-intravenous acyclovir treatment (B) along with the ulnar nerve distribution with a claw-like deformity of the right hand. 


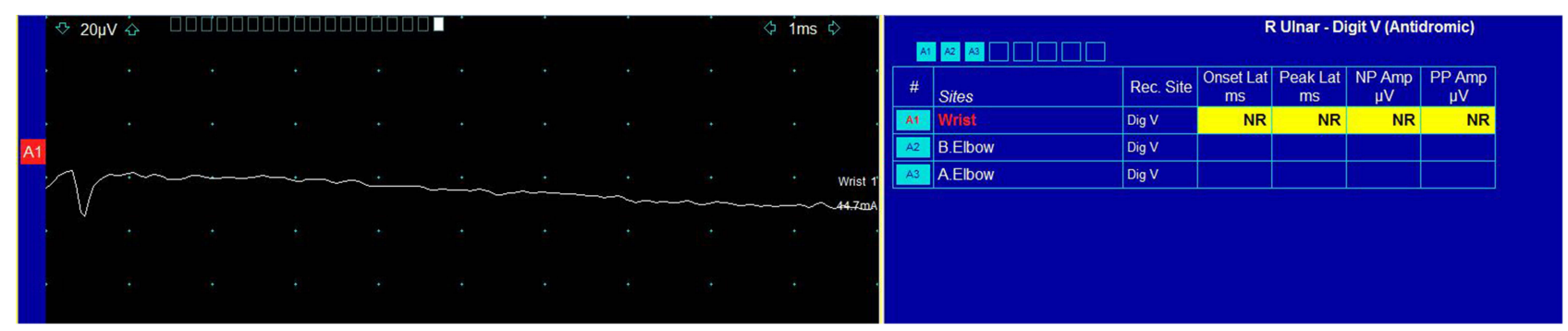

Figure 2 Right ulnar sensory nerve conduction study (NCS) showed no response (yellow highlighting).

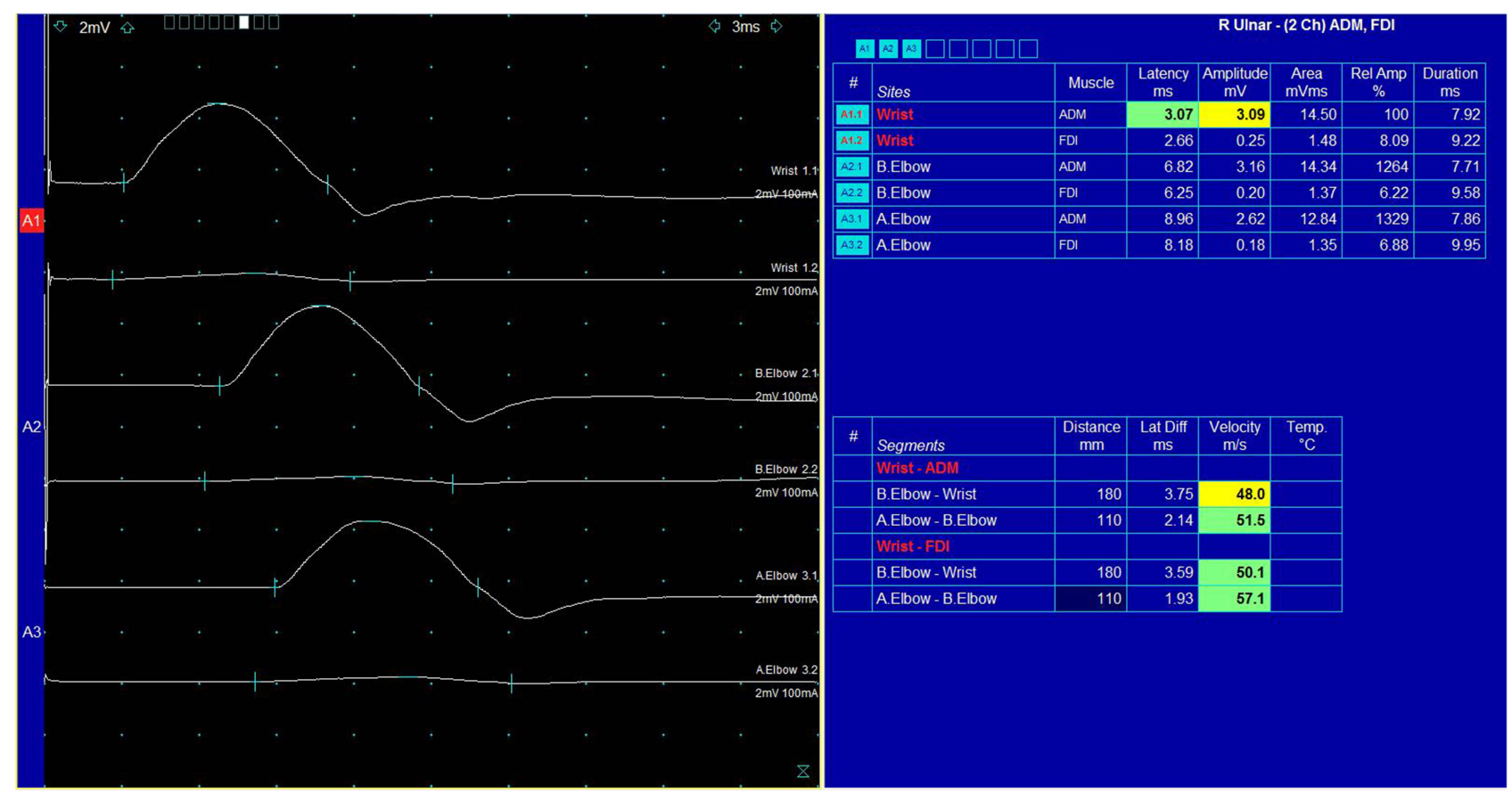

Figure 3 Right 2-channel ulnar motor nerve conduction study (NCS) recorded at the abductor digiti minimi (ADM) and first dorsal interosseous (FDI) muscles, respectively. This demonstrates decreased ADM compound motor action potential (CMAP) amplitude (yellow highlighting) and severely decreased FDI CMAP amplitude (normal ulnar CMAP amplitude in our laboratory is $\geq 5.0 \mathrm{mV}$ ) with a slight decrease in motor nerve conduction velocity (NCV) (yellow highlighting), which can be concluded as axonal involvement of motor fibers, more severe in FDI than ADM muscles.
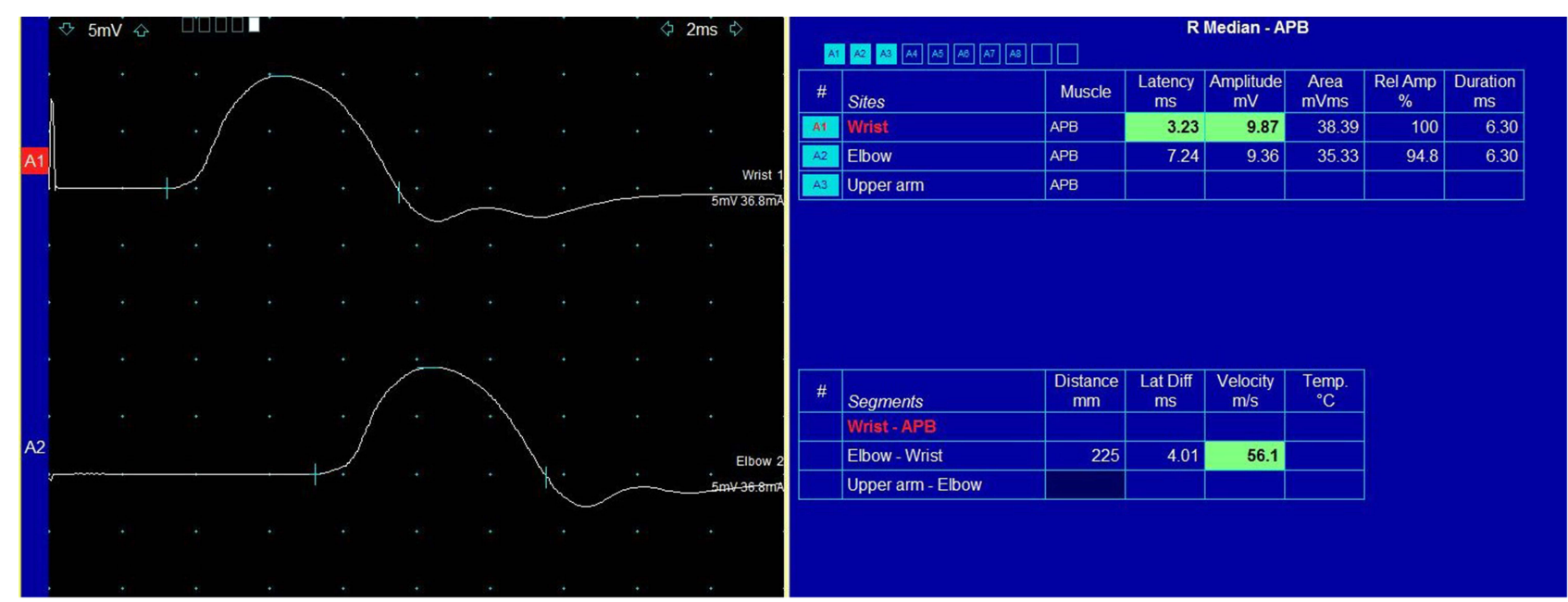

Figure 4 Right median motor nerve conduction study (NCS) recording at abductor pollicis brevis (APB) muscle demonstrated normal compound motor action potential (CMAP) amplitude (normal median CMAP amplitude in our laboratory is $\geq 5.0 \mathrm{mV}$ ) with normal motor nerve conduction velocity (NCV) (green highlighting). 
instability in right FDI, ADM, fourth and fifth flexor digitorum profundus (FDP), and APB muscles, obviously in the FDI muscle, but normal in right flexor pollicis longus (FPL), flexor digitorum superficialis (FDS), extensor indicis proprius (EIP), extensor carpi radialis (ECR), biceps brachii, triceps brachii including cervical paraspinal muscles. Thus, the electrodiagnostic finding was compatible with right multiple mononeuropathies of the median and ulnar nerves.

As a right-handed person, impairment of right intrinsic hand muscle strength affected her job tasks and daily-life activities. The right fingers were clawing with loss of synchronistic finger flexion with difficulty digit abduction and adduction resulting in weakness of the right handgrip. For initial correction of this deformity, the MCP joints need to be flexed beyond neutral to produce enough tension to the extensor tendon for IP joint extension. ${ }^{10}$ We prescribed occupational therapy treatment including kinetic activities with fine motor training of the right hand and a static hand splint for MCP joint flexion, as shown in Figure 5, which facilitated synchronized finger flexion to help the patient return to her work using a keyboard for typing.

The patient was also given physical therapy programs including electrical stimulation to the atrophic muscles (right FDI and ADM muscles) with the intrinsic hand strengthening technique. The static splint was removed after 4 months of use by the patient because of a stronger right handgrip. At 7 months after the infection with full medical and rehabilitation treatment, the patient felt some remaining paresthesia along with right ulnar nerve distribution without pain. She felt a stronger right handgrip, which corresponded to the improvement of muscle power in the right hand by a physical examination, which was grade III for the right FDI muscle, grade IV for the right ADM muscle, and grade $\mathrm{V}$ for the right APB muscle. The grip strength of the right hand was 6.3 kilograms, which improved to approximately 5 kilograms compared with the examination 7 months previously. The repeated electrodiagnostic study revealed significant improvement of both sensory and motor nerve conduction studies of the right ulnar nerve. There was no functional limitation but mild poor endurance of the right intrinsic hand muscles by clenched fist activities, such as wringing out a towel. There was no persistent wound scar or stiffness of the right hand.

\section{Discussion}

Generally, HZ attacks one or two adjacent thoracic and lumbar dermatomes, as well as the cranial nerve distribution. The infection is characterized by unilateral painful maculopapular rash transformed to vesicles or bullae along the dermatomal distribution. ${ }^{1,2}$ Myotomes corresponding to the dermatomal distribution can occasionally be attacked by HZ, causing weakness of the involved extremity. It typically occurs a few weeks after the cutaneous symptom. ${ }^{5,13}$ Radiculopathy is a common form of HZrelated weakness that mainly involves the proximal upper extremity (C5-7 distribution). ${ }^{11,12}$ Weakness of the distal upper extremity (C8-T1 distribution) is rare and can be

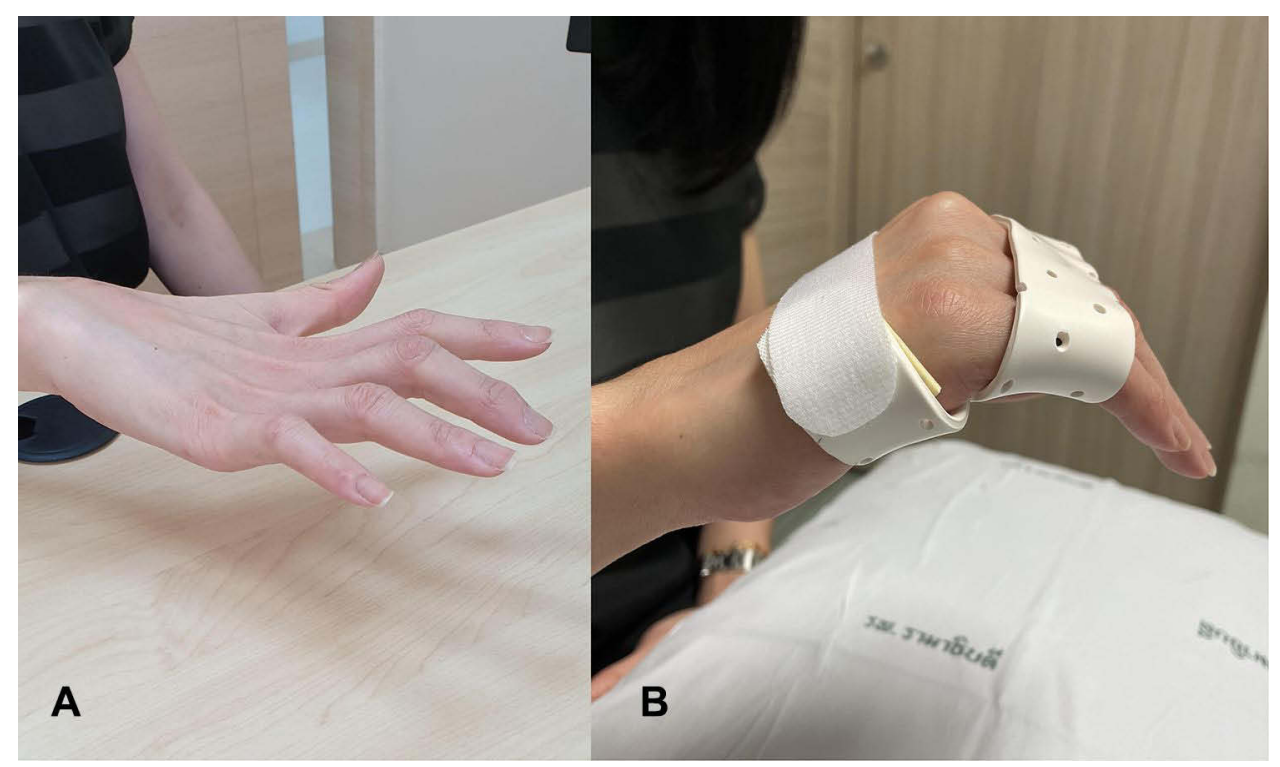

Figure 5 Right claw hand deformity (metacarpophalangeal (MCP) joint hyperextension and interphalangeal (IP) joint slightly flexion) in resting position (A) and after wearing a static figure-of-eight splint for MCP joint flexion (B). 
confused with nerve entrapment syndrome such as carpal and cubital tunnel syndromes. ${ }^{13}$ Moreover, HZ-related mononeuropathy, especially in the distal upper extremity, is uncommon by individual reports. Previous studies of distal upper extremity mononeuropathy in mixed sensorymotor nerves after $\mathrm{HZ}$ described the clinical presentation as compatible with our patient. A cutaneous lesion associated with sharp shooting pain along suspected nerve distribution occurred earlier followed by motor weakness and loss of hand function. ${ }^{6-9,14}$ The most common causes of $\mathrm{HZ}$ are immunosuppression and immunity-declined elderly. ${ }^{1-3}$

We reported on an immunocompromised 31-year-old woman with multiple mononeuropathies of the right ulnar and median nerves after zoster reactivation. The electrodiagnostic results of HZ-related weakness patients from the literature revealed reduced or absent sensory nerve action potential (SNAP), reduced CMAP amplitudes, while latency and NCV were mildly affected by drop out of the fastest axon, unlike the result from nerve entrapment syndrome that usually presents as conduction block and affected NCV. Ongoing axonal loss by electrodiagnosis was also observed in our patient, which corresponded to previous studies. $^{13,15,16}$

Regarding DLBCL, a type of non-Hodgkin lymphoma (NHL), the tumor can infiltrate the peripheral nerves causing axonal damage by patterns of generalized neuropathy, plexopathy, or even mononeuropathy, but with lower incidence than central complications. Such neuropathies are categorized into axonal and demyelinating involvements as well as symmetrical and asymmetrical types that can be distinguished by electrodiagnostic study. The axonal type is the most frequent type and is often due to chemotherapy. ${ }^{17,18}$

The most suspected chemotherapy drug treated in our patient is vincristine. A common dose-limiting side effect of vincristine is neurotoxicity. ${ }^{19}$ Clinically, chemotherapyinduced peripheral neuropathy (CIPN) usually presents as deficits in sensory, motor, and autonomic function which develop in a glove and stocking pattern due to preferential effects on longer axons (so-called length-dependent neuropathy), unlike the neuropathic pattern that occurred in our patient. $^{20}$

Prognosis in functional motor recovery after $\mathrm{HZ}$ is considered good in about $75 \%$ of the cases, but may take months to years. ${ }^{15}$ Early diagnosis of $\mathrm{HZ}$ and early administration of antiviral therapy (acyclovir) has been proven to reduce the viral spread and therefore prevent and minimize both sensory and motor involvement. ${ }^{21}$ Recently, a nonliving vaccine is believed to be an option for the prevention of herpes zoster in individuals who are immunocompromised. ${ }^{22}$ In this case, intrinsic hand paralysis is the outcome of the infection. Occupational therapy was the preliminary choice for our patient. We prescribed the static figure-of-eight splint in flexion position for the right MCP joint. Thus, she could use her dominant hand to do her job and daily-life activities while we waited for her nerves to recover. The surgical treatment option may be the future choice if functional motor recovery of the intrinsic hand muscles is not regained sufficiently.

\section{Conclusion}

HZ-related distal mononeuropathy is a rare complication, especially in the upper extremity. Early recognition and early electrodiagnosis are necessary to exclude nerve entrapment syndrome and to be the baseline for functional motor recovery prediction. Misdiagnosis as severe nerve entrapment syndrome could lead to improper surgical management, which could result in a worsened condition. The prognosis for recovery is considered good but may take months to years to accomplish. A claw-like deformity should be corrected preliminarily by a hand splint, which is then ceased after improvement of hand function.

\section{Ethic and Consent Statement}

This case report was approved by the Ethics Committee on Human Rights related to research involving human subjects at Faculty of Medicine Ramathibodi Hospital. The written informed consent was obtained from the patient for publication of this case report and any accompanying images.

\section{Disclosure}

The authors declare that they have no conflicts of interest.

\section{References}

1. Weaver BA. Herpes zoster overview: natural history and incidence. J Am Osteopath Assoc. 2009;109:2-6.

2. Hope-Simpson RE. The nature of herpes zoster: a long-term study and a new hypothesis. Proc R Soc Med. 1965;58:9-20.

3. Gnann JW, Whitley RJ. Clinical practice: herpes zoster. $N$ Engl J Med. 2002;347:340-346. doi:10.1056/NEJMcp013211

4. Saguil A, Kane S, Mercado M, Lauters R. Herpes zoster and postherpetic neuralgia: prevention and management. Am Fam Physician. 2017;96:656-663.

5. Yoleri O, Olmez N, Oztura I, Sengül I, Günaydin R, Memiş A. Segmental zoster paresis of the upper extremity: a case report. Arch Phys Med Rehabil. 2005;86:1492-1494. doi:10.1016/j.apmr.20 04.09.032 
6. Kayipmaz M, Basaran SH, Ercin E, Kural C. Isolated ulnar dorsal cutaneous nerve herpes zoster reactivation. Orthopedics. 2013;36:1217-1219. doi:10.3928/01477447-20130821-28

7. Athwal GS, Bartsich SA, Weiland AJ. Herpes zoster in the ulnar nerve distribution. J Hand Surg Br. 2005;30:355-357. doi:10.1016/J. JHSB.2005.04.010

8. Matondo P, Lungu G, Njobvu P. Claw hand as a complication of herpes zoster. Trop Doct. 2000;30:33-35. doi:10.1177/ 004947550003000118

9. Tahiri Y, Hamelin ND, Brutus JP. Herpes zoster in the median nerve distribution. J Plast Reconstr Aesthet Surg. 2010;63:195-196. doi:10.1016/j.bjps.2009.06.014

10. Sapienza A, Green S. Correction of the claw hand. Hand Clin. 2012;28:53-66. doi:10.1016/j.hcl.2011.09.009

11. Claflin B, Thomas M, Wilson AJ. Polyradiculopathy and herpes zoster. Proc (Bayl Univ Med Cent). 2009;22:223-225. doi:10.1080/ 08998280.2009.11928521

12. Braverman DL, Ku A, Nagler W. Herpes zoster polyradiculopathy. Arc Phys Med Rehabil. 1997;78:880-882. doi:10.1016/S00039993(97)90204-5

13. Kreps CE, Rynders SD, Chhabra AB, Jenkins JG. C8 myotome herpes zoster paresis. Am J Orthop (Belle Mead NJ). 2012;41:220-222.

14. Reda H, Watson JC, Jones LK. Zoster-associated mononeuropathies (ZAMs): a retrospective series. Muscle Nerve. 2012;45:734-739. doi:10.1002/mus.23342
15. Merchut MP, Gruener G. Segmental zoster paresis of limbs. Electromyogr Clin Neurophysiol. 1996;36:369-375.

16. Jones LK, Reda H, Watson JC. Clinical, electrophysiologic, and imaging features of zoster-associated limb paresis. Muscle Nerve. 2014;50:177-185. doi:10.1002/mus.24141

17. Kelly JJ, Karcher DS. Lymphoma and peripheral neuropathy: a clinical review. Muscle Nerve. 2005;31:301-313. doi:10.1002/ mus. 20163

18. Grisold W, Grisold A, Marosi C, Meng S, Briani C. Neuropathies associated with lymphoma. Neurooncol Pract. 2015;2:167-178.

19. Madsen ML, Due H, Ejskjær N, Jensen P, Madsen J, Dybkær K. Aspects of vincristine-induced neuropathy in hematologic malignancies: a systematic review. Cancer Chemother Pharmacol. 2019;84:471-485. doi:10.1007/s00280-019-03884-5

20. Starobova H, Vetter I. Pathophysiology of chemotherapy-induced peripheral neuropathy. Front Mol Neurosci. 2017;10:174. doi:10.3389/fnmol.2017.00174

21. Mondelli M, Romano C, Passero S, Porta PD, Rossi A. Effects of Acyclovir on sensory axonal neuropathy, segmental motor paresis and postherpetic neuralgia in herpes zoster patients. Eur Neurol. 1996;36:288-292. doi:10.1159/000117274

22. Warren-Gash C, Breuer J. Herpes zoster in people who are immunocompromised: what are the options for prevention? Lancet Infect Dis. 2019;19:922-924. doi:10.1016/S1473-3099(19)30399-8
Orthopedic Research and Reviews

\section{Publish your work in this journal}

Orthopedic Research and Reviews is an international, peer-reviewed, open access journal that focusing on the patho-physiology of the musculoskeletal system, trauma, surgery and other corrective interventions to restore mobility and function. Advances in new technologies, materials, techniques and pharmacological agents are particularly

\section{Dovepress}

welcome. The manuscript management system is completely online and includes a very quick and fair peer-review system, which is all easy to use. Visit http://www.dovepress.com/testimonials.php to read real quotes from published authors. 